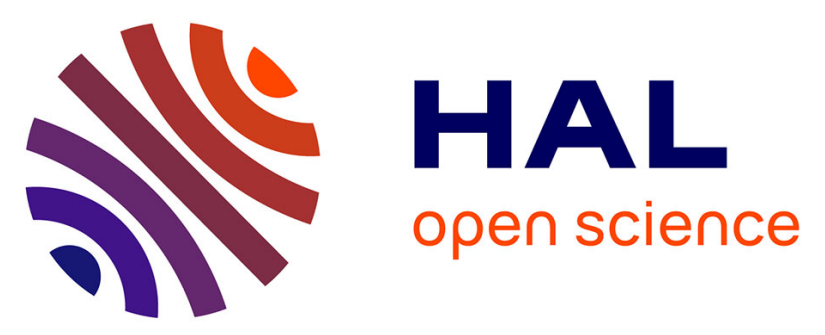

\title{
The role of auditory feedback in speech development: A study of compensation strategies for a lip-tube perturbation
}

\author{
Lucie Ménard, Pascal Perrier, Jérôme Aubin
}

\section{To cite this version:}

Lucie Ménard, Pascal Perrier, Jérôme Aubin. The role of auditory feedback in speech development: A study of compensation strategies for a lip-tube perturbation. ICA 2013 - Acoustics 2013 - 21st International Congress on Acoustics - 165th Meeting of the Acoustical Society of America, Jun 2013, Montréal, Canada. Paper 4pSCa6. hal-00847186

\section{HAL Id: hal-00847186 \\ https://hal.science/hal-00847186}

Submitted on 23 Jul 2013

HAL is a multi-disciplinary open access archive for the deposit and dissemination of scientific research documents, whether they are published or not. The documents may come from teaching and research institutions in France or abroad, or from public or private research centers.
L'archive ouverte pluridisciplinaire HAL, est destinée au dépôt et à la diffusion de documents scientifiques de niveau recherche, publiés ou non, émanant des établissements d'enseignement et de recherche français ou étrangers, des laboratoires publics ou privés. 


\section{Proceedings of Meetings on Acoustics}
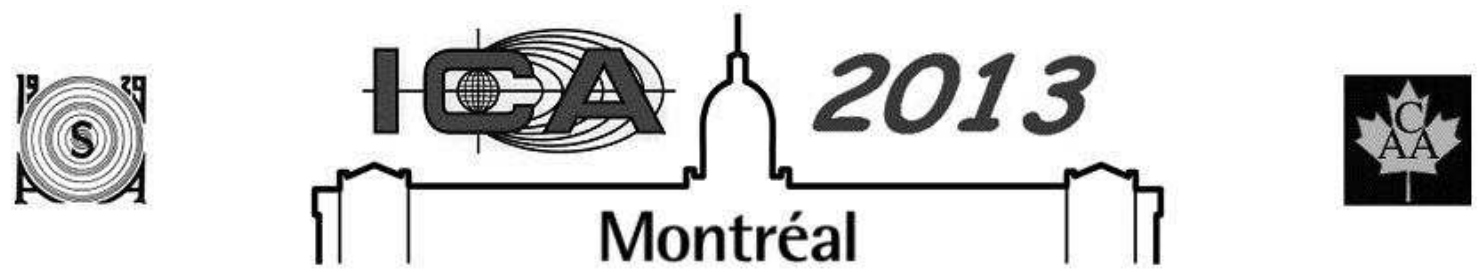

ICA 2013 Montreal

Montreal, Canada

2 - 7 June 2013

Speech Communication

Session 4pSCa: Auditory Feedback in Speech Production II

\section{4pSCa6. The role of auditory feedback in speech development: A study of compensation strategies for a lip-tube perturbation}

Lucie Menard*, Pascal Perrier and Jérôme Aubin

*Corresponding author's address: Linguistics, Université du Québec à Montréal, CP 8888, succ. Centre-Ville, Montreal, H3C 3P8, Quebec, Canada, menard.lucie@uqam.ca

The role of auditory feedback in speech development was investigated through a study of compensation strategies for a lip-tube perturbation. Acoustic, articulatory, and perceptual analyses of the vowels /i/, /y/, and /u/ produced by ten 4-year-old French speakers and ten adult French speakers were conducted under two conditions: normal and with a 15-mm-diameter tube (for /y/ and /u/) or a 5-mm-diameter tube (for /i/) inserted between the lips. Ultrasound and acoustic recordings of isolated vowels were made in normal condition before any perturbation (N1), for each of the 20 trials in the perturbed condition (P), and in normal condition after the perturbed trials (N2). Data reveal that adult participants moved their tongue in the P condition more than children subjects, to compensate for F1 and F2 alteration induced by the tube. Except for /y/, the perturbation was generally at least partly compensated during the perturbed trials in adults and children, but children did not show a typical learning effect. Results are analyzed from the perspective of (i) goal specification in speech production in the acoustic and/or somatosensory domain, and (ii) the maturity of representations of the motor apparatus in the brain.

Published by the Acoustical Society of America through the American Institute of Physics 


\section{INTRODUCTION}

Perturbation experiments have been widely used to study articulatory compensation, a capacity which enables the speech production system to achieve a goal, even if this system is constrained in some way (Lindblom and Sundberg, 1971). Artificial perturbations such as artificial palates or lip-tubes have the advantage of modifying important geometric characteristics of the vocal tract, and hence constraining speakers to develop new strategies to produce the intended sound. The lip-tube is an appropriate mechanism to affect the geometry of the vocal tract as it increases or decreases the lip area over that found in the typical configuration. In the French vowel /u/, for instance, the lips are rounded so that the lip area is small (around $0.3 \mathrm{~cm}^{2}$ for adults, Savariaux et al., 1995); the associated tongue position is elevated towards the velopalatal region. It has been found that, when a 2.5 -cm-diameter lip-tube keeps them from rounding their lips, French adult speakers required to produce $/ \mathrm{u} /$ cannot immediately compensate for the perturbation. After a learning session, during which a constant improvement of the / $\mathrm{u} /$ quality was observed, some speakers were better able to compensate than others, and their compensation strategies involved a backward movement of the tongue towards the velo-pharyngeal region (Savariaux et al., 1995, 1999). More recent studies using a lip-tube with children (Ménard et al., 2008) showed that 4-year-old children could successfully compensate for a lip-tube perturbation (as assessed by the intelligibility of their produced vowels) but that the compensation process was based on trial and error, rather than on a real error correction mechanism. However, both children and adults showed a significant improvement in their production when the lip-tube was removed compared to the normal condition, suggesting some recalibration of their acoustic-articulatory models. How do these models differ between children and adults?

In this paper, we report on an experiment in which the acoustic and articulatory effects of labial perturbations on speech produced by 4-year-old children and adult French speakers were assessed. Articulatory data were collected using an ultrasound system, a non-invasive technology that allows imaging of the tongue surface (Stone, 2005). A perceptual analysis of acoustic data was carried out to determine the efficiency of the articulatory strategies applied. Two points of view - production and perception - will be examined to shed light on compensation processes from a developmental perspective.

\section{METHOD}

\subsection{Subjects}

Two groups of Quebec French speakers participated in this experiment. The first one, referred to as the "child group," consisted of ten children (four males and six females) averaging 4 years and 2 months in age (from 3 years 10 months to 4 years 11 months). The second group, referred to as the "adult group," consisted of ten adults (three males and seven females). They ranged in age from 21 to 29 years old, with a mean age of 24 years. A screening procedure ensured that all subjects were free of any speech, hearing or phonological disorder.

\subsection{Stimuli}

In addition to the vowel $/ \mathrm{u} /$, which was the one used in previous lip-tube experiments, the French vowels $/ \mathrm{i} /$ and $/ y /$ were used in this corpus. Only results for the rounded vowels $/ y /$ and $/ \mathrm{u} /$ are reported here. Twenty repetitions of each vowel were produced in isolation by each speaker in each of the following conditions: before the insertion of the lip-tube (N1), with the tube in place (P), and immediately after removal of the tube (N2). The order of the three conditions was the same for each vowel (N1, P, N2). Vowel order was randomized across subjects.

\subsection{Production experiment}

Lip-tubes were used to create a greater lip area while speakers pronounced the rounded vowels $/ \mathrm{u} / \mathrm{and} / \mathrm{y} /$. The tubes were made of fine layers of plastic and had diameters of $1.5 \mathrm{~cm}$ and $2 \mathrm{~cm}$, respectively, for children and adults. Several simulations using an articulatory-to-acoustic model of the growing vocal tract (Variable Linear Articulatory Model, developed by S. Maeda, have revealed that those diameters were well adapted for those speaker groups (Aubin, 2005). Results of those simulations are displayed in Figures 1 and 2. The predicted acoustic changes upon insertion of the tube (increased lip area) without any compensation were as follows. For the vowel $/ \mathrm{y} /$, insertion of 
the tube yielded an increase in F2 and F3 values (and to a lesser extent, F1) for both speaker groups (and to a lesser extent, F1). For /u/, insertion of the tube yielded an increase in F1 (more so for children than for adults) and F2 values.
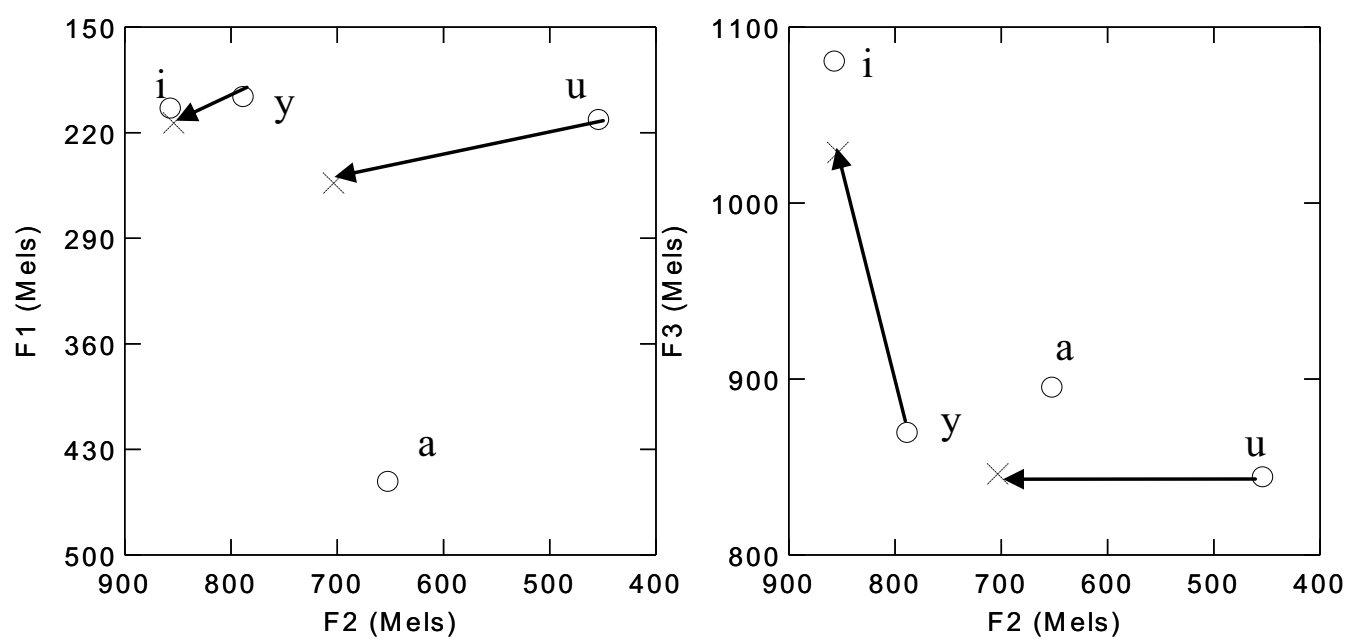

FIGURE 1. Simulations of a lip-tube perturbation of $/ y /$ and $/ u$ / with Maeda's articulatory-to-acoustic model simulating growth for the adult stage. Normal, unperturbed $/ \mathrm{i} /, / \mathrm{y} /, \mathrm{u} /$, and /a/ vowels are depicted by black circles, and perturbed /y/ and /u/ without any compensation are shown by black crosses. For the sake of clarity, normal and perturbed vowels are linked by a black arrow (pointing towards the perturbed one).
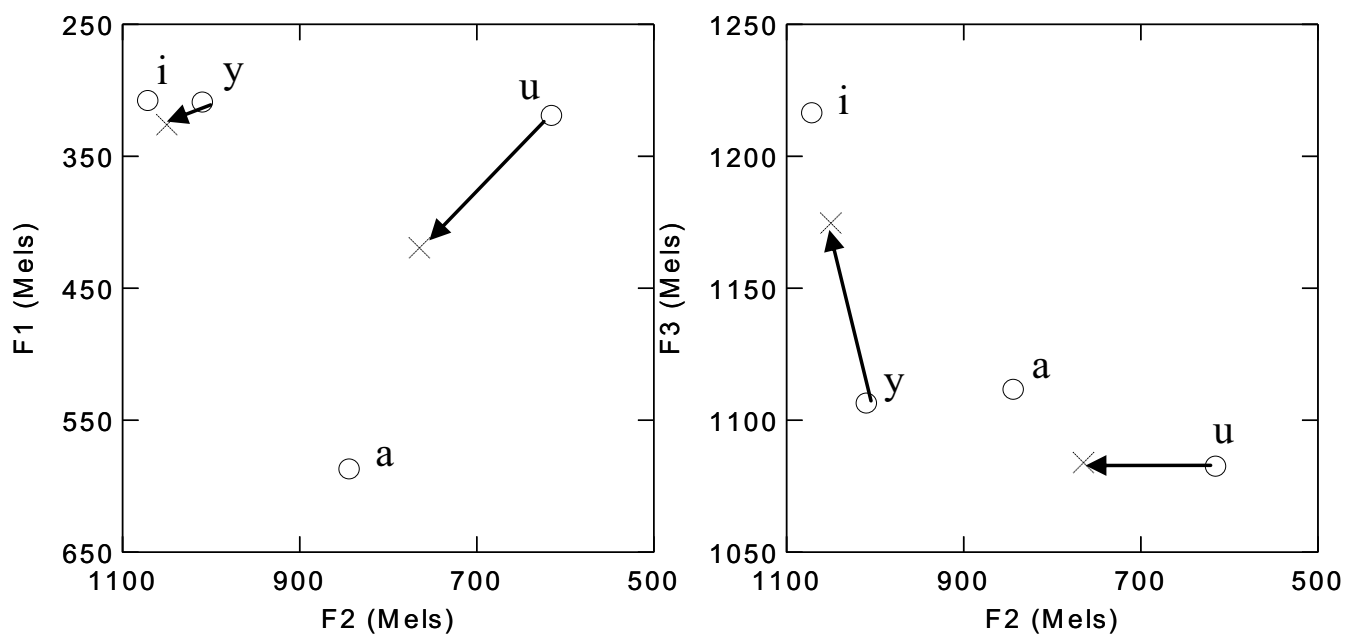

FIGURE 2. Simulations of a lip-tube perturbation of $/ y /$ and $/ \mathrm{u} /$ with Maeda's articulatory-to-acoustic model simulating growth for the 4-year-old stage. Normal, unperturbed /i/, /y/, /u/, and /a/ vowels are depicted by black circles, and perturbed /y/ and /u/ without any compensation are shown by black crosses. For the sake of clarity, normal an perturbed vowels are linked by a black arrow (pointing towards the perturbed one).

Lip-tube lengths were adjusted for each speaker to prevent lengthening of the oral cavity. The subjects were seated comfortably in a quiet room. Articulatory data were obtained using a Sonosite 180 Plus ultrasound system. The system's transducer (84-degree curved array) was attached to a microphone stand and was held still, with the speaker's head. The acoustic signal was captured by an unidirectional microphone. Both ultrasound and microphone signals were recorded by a MiniDV Panasonic AG-DVC 30 camcorder, in NTSC format. 


\subsection{Perception experiment}

A subset of the produced corpus served as stimuli for a perceptual experiment. In each of the normal experimental conditions (N1, N2), only repetitions 1, 2, and 3 were selected for the listening experiment. In the perturbed condition, repetitions $1,3,5,7,9,11,13,15,17,19$, and 20 were selected. Stimuli were presented once via high-quality headphones in a blocked condition by speaker. Within a block, each speaker's stimuli were randomized and block order was randomized as well. Twenty French listeners (10 males, 10 females) aged from 19 to 25 years old participated in this experiment. Listeners had to identify the vowel they perceived and to evaluate its quality on a scale ranging from 1 (bad) to 5 (excellent).

\subsection{Data analysis}

\section{Production data}

The audio signals were labeled and vowels were extracted using Praat. Fundamental frequency and formant values were extracted at vowel midpoint. The acoustic signal was downsampled to $22 \mathrm{KHz}$ after low-pass filtering (cut-off frequency of $10 \mathrm{KHz}$ ) and an LPC analysis detected up to four formants (the number of poles ranged from 12 to 18). Fundamental frequency measurements were made using the autocorrelation method. Formant and F0 values were then transformed into the Mel scale. Each acoustic parameter (F1, F2, F3, F0) in perturbed condition was measured as a proportion of the same parameter under unperturbed condition. For each speaker, the ratio of the acoustic values in the perturbed condition relative to the acoustic values in the normal pre-perturbed condition was computed (values in P / values in N1). Similar ratios were computed for the acoustic values in the N2 condition (values in $\mathrm{N} 2$ / values in N1). Using this method, acoustic data were transformed to within-speaker normalized ratios relative to N1. Separate repeated-measures ANOVAs were conducted on F1, F2, F3, or F0 as the dependent variables; speaker group (children and adults) as the between-subject factor; and vowel $(/ \mathrm{u} /$ and $/ \mathrm{y} /$ ) and condition (perturbed P and post-perturbed N2) as the within-subject factors.

Ultrasound sagittal images corresponding to vowel midpoint were extracted using Adobe Premiere Pro. Tongue surface contours were measured using EdgeTrak, a semiautomatic system dedicated to this purpose (Li et al., 2003). The 100-point contours were exported to an in-house Matlab application that extracts several parameters quantifying tongue contours (Ménard et al., 2012). For each tongue contour, the $\mathrm{x}$ and y coordinates of the contour's highest point (on the vertical axis) were extracted. Note that this point can be interpreted as an absolute measure of tongue height in reference to the probe but does not correspond to a measure of tongue distance relative to the palate. These two parameters allowed the characterization of the articulatory space along the tongue height and tongue frontness parameters. Tongue contours across experimental conditions were compared along those dimensions. Average backward displacement of the tongue relative to $\mathrm{N} 1$ was calculated for vowels in the P and $\mathrm{N} 2$ condition, in mm. Separate repeated-measures ANOVAs were conducted with $x$ or $y$ values of the highest point of the tongue as the dependent variables; speaker group (children and adults) as the between-subject factor; and vowel (/u/ and $/ \mathrm{y} /$ ) and condition (perturbed P and normal post-perturbed N2) as the within-subject factors.

\section{Perceptual data}

To assess the efficiency of the articulatory-acoustic strategies, the perceptual scores were computed as follows. For each vowel and in each condition, the mean (across the 20 listeners) percent correct identification of the vowel weighted by the quality rating score was obtained (number of correct responses * quality rating score). Perceptual scores were then transformed into within-speaker normalized ratios relative to N1 using the method described in the previous paragraph for the acoustic data. Note that for this preliminary analysis, perceptual data were averaged across all stimuli in a given condition. A repeated-measures ANOVA was conducted with perceptual scores as the dependent variable, speaker group (children and adults) as the between-subject factor, and vowel (/u/ and $/ \mathrm{y} /$ ) and condition (perturbed $\mathrm{P}$ and normal post-perturbed N2) as the within-subject factors.

In the perturbed condition $\mathrm{P}$, a vowel is considered totally compensated for if its perceptual score is equal to (or greater than) the perceptual score in the normal pre-perturbed condition P1. A perturbed vowel (P) for which the perceptual score is lower than the score in the normal pre perturbed condition N1 can result from (i) compensation strategies that were not totally successful or (ii) the absence of any compensation. Acoustics and articulatory data will be examined to explore both possibilities. 


\section{RESULTS}

\subsection{Perception}

The results of the perceptual task are shown in Figure 3, for the perturbed condition $\mathrm{P}$ and for the postperturbation N2 condition. Recall that the data are perceptual scores relative to the normal pre-perturbation condition N1. Thus, values greater than 1 (above the dashed line) correspond to vowels for which perception was improved compared to N1, whereas values lower than 1 (below the dashed line) correspond to vowels for which perception was degraded compared to N1. Data are shown separately for each speaker group (adults: solid red line; children: dotted blue line), and for each vowel (/y/: left panel; /u/: right panel).
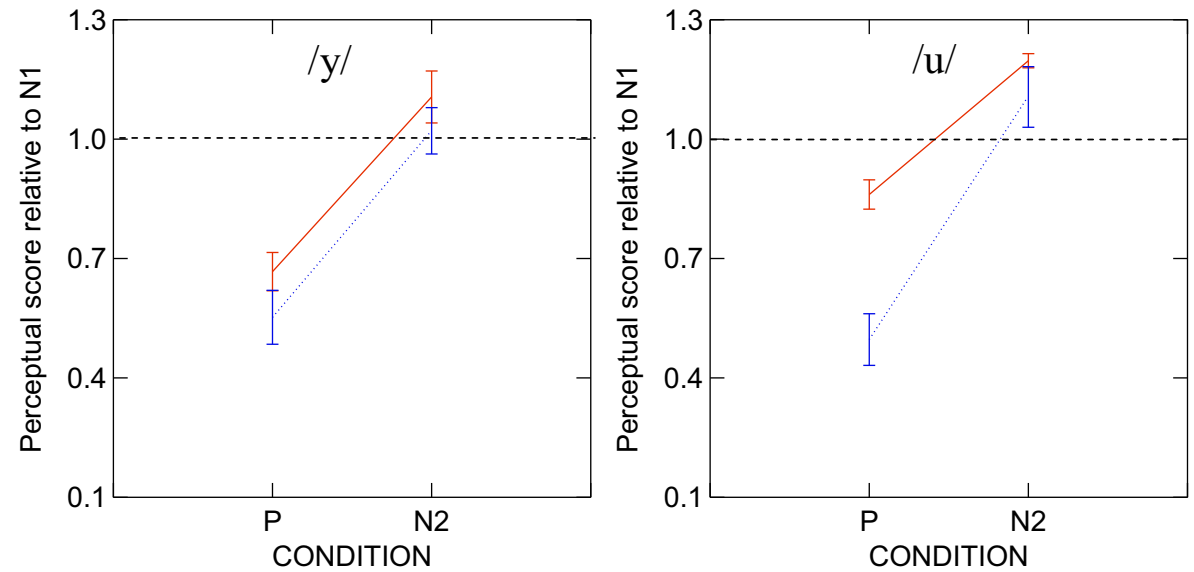
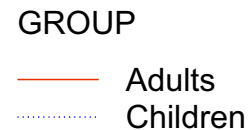

FIGURE 3. Perceptual score relative to the normal pre-perturbed condition N1, averaged across speaker groups, for the perturbed condition $\mathrm{P}$ and the normal post-perturbed condition N2. Adult data correspond to the solid red line and child data to the dotted blue line. Data are shown separately for $/ \mathrm{y} /$ (left panel) and /u/ (right panel).

Figure 1 shows that, overall, adult speakers produced vowels that were associated with higher perceptual scores than children $(F(1,18)=9.74 ; \mathrm{p}<.05)$. The results of the repeated-measures ANOVA revealed a significant effect of condition on the perceptual scores. For both vowels, perceptual scores in the perturbed condition were lower than the perceptual scores in the normal pre-perturbed condition N1 (scores $<1$ ), indicating that participants were not totally successful in their attempts to compensate for the perturbation $(F(1,18)=1083.53 ; \mathrm{p}<.001)$. However, all participants produced vowels in the post-perturbed condition N2 that were associated with higher perceptual scores than in the normal pre-perturbed condition N1 (scores $>1$ ). Thus, despite the degraded perceptual quality of the perturbed vowels, participants seemed to have applied some compensation strategies, leading to modifications in their articulatory-to-acoustic relationships. This issue will be discussed further below, together with the articulatory and acoustic data. When examining data for each vowel, it can be noted that adult participants produced /y/ vowels with higher perceptual scores than children participants (significant interaction of vowel and group factors $(\mathrm{F}(1,32)=598.19 ; \mathrm{p}<.001))$. As for $/ \mathrm{u} /$, adults were more successful than children in the perturbed condition, as shown by their higher perceptual score in this condition. In the post-perturbed condition N2, they also produced /u/ vowels that were associated with higher perceptual scores than children, but there was less difference between age groups than in the perturbed condition P.

\subsection{Production}

Turning now to the production data, acoustic values will be examined first. Ratios of F1 values in the P and N2 conditions relative to the N1 condition are shown in Figure 4 for both speaker groups and both vowels. 

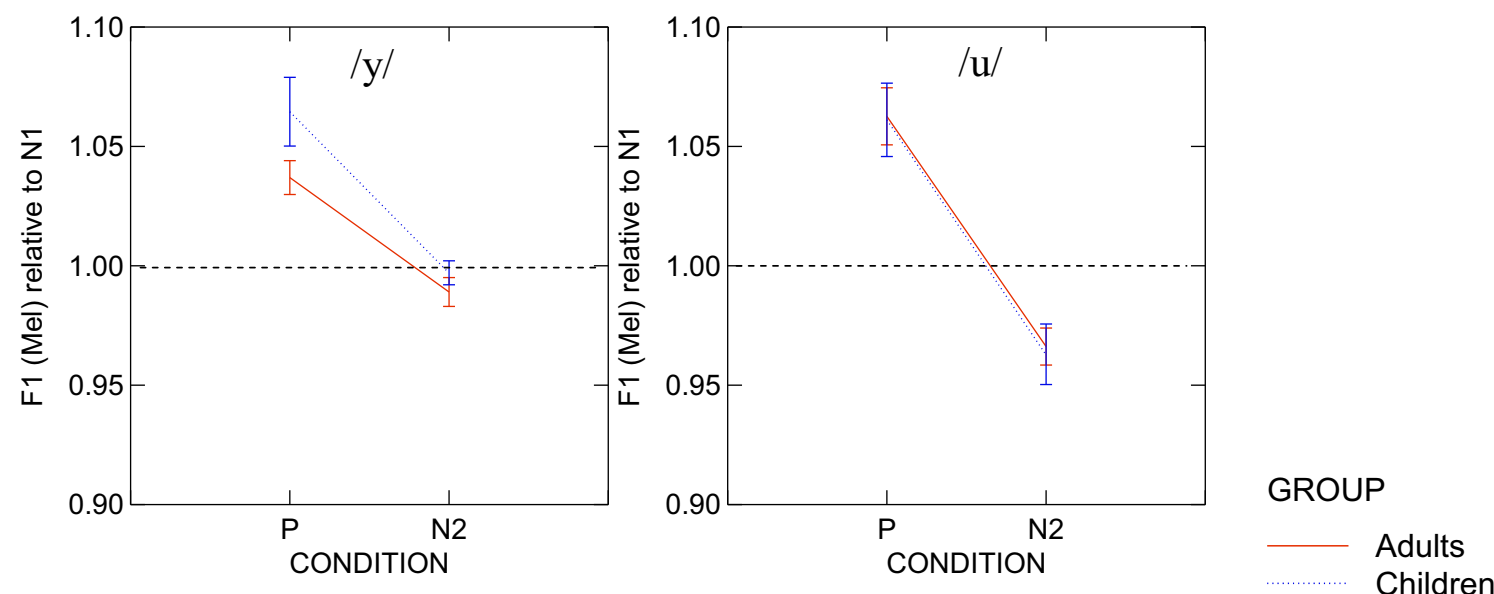

FIGURE 4. F1 values (in Mel) relative to the normal pre-perturbed condition N1, averaged across speaker groups, for the perturbed condition $\mathrm{P}$ and the normal post-perturbed condition N2. Adult data correspond to the solid red line and child data to the dotted blue line. Data are shown separately for $/ \mathrm{y} /$ (left panel) and /u/ (right panel).

Figure 4 shows that for both speaker groups and for both vowels, F1 ratios were greater than 1 in the perturbed condition $\mathrm{P}$, revealing that speakers did not fully compensate for the lip-tube perturbation in this acoustic dimension. A strong after-effect is revealed for adults: relative F1 values were lower than 1 in the post-perturbed condition N2 (thus, lower than in the normal pre-perturbed condition N1) for $/ \mathrm{y} /$ and $/ \mathrm{u} /(\mathrm{F}(1,18)=108.24 ; \mathrm{p}<.001)$. For children, $\mathrm{F} 1$ values were also lower in N2 compared to the $\mathrm{P}$ and $\mathrm{N} 1$ conditions, but this pattern in the $\mathrm{N} 2$ condition is observed only for $/ \mathrm{u} /$. Indeed, for $/ \mathrm{y} /$, relative $\mathrm{F} 1$ values in $\mathrm{N} 2$ are close to 1 , suggesting that there was no change in F1 compared to the normal pre-perturbed condition (N1). Recall, however, that according to the simulations (Figures 1 and 2), F1 was not much affected by a lip-tube for $/ \mathrm{y} /$. Thus, observed changes in Figure 4 for $/ \mathrm{y} / \mathrm{might}$ be an artifact of other strategies aimed at lowering F2 and / or F3.

F2 values relative to N1 are depicted in Figure 5, for both speaker groups and for both vowels, across conditions. Different patterns can be observed for the two vowels. For /y/, F2 values relative to N1 were significantly lower for children than for adults $(\mathrm{F}(1,18)=24.20 ; \mathrm{p}<.01)$, in both conditions $(\mathrm{P}$ and $\mathrm{N} 2)$. Thus, children reduced their $\mathrm{F} 2$ values with the lip-tube in place, probably to compensate for the increased F2 values induced by the tube. This strategy was preserved after removal of the tube, as revealed by the F2 values lower than 1 in the N2 condition. In adult subjects, the lip-tube did not alter F2 to the same extent, resulting in F2 values for $/ \mathrm{y} /$ after removal of the tube that were similar $(=1)$ to the values in the $\mathrm{N} 1$ condition. Recall, however, that the /y/ vowels produced by adults in the N2 condition had better perceptual scores than those produced in the N1 condition (scores $>1$, see Figure 1). The F2 dimension for $/ y /$ thus seems to be less related to the perceptual goal for this group.

For the vowel $/ \mathrm{u} /$, adults and children had increased values of F2 in the perturbed condition $\mathrm{P}$ compared to the $\mathrm{N} 1$ (ratios $>1$ ) and $\mathrm{N} 2$ conditions $(\mathrm{F}(1,18)=230.42 ; \mathrm{p}<.001)$. Although they did not totally compensate acoustically for the insertion of the lip-tube (as shown by the ratio values greater than 1 in the P condition), they altered their articulatory-acoustic model, as their F2 values are significantly lower in the N2 condition. Adults performed better than children, with their relative F2 values being less increased in the perturbed condition P than the children's. 

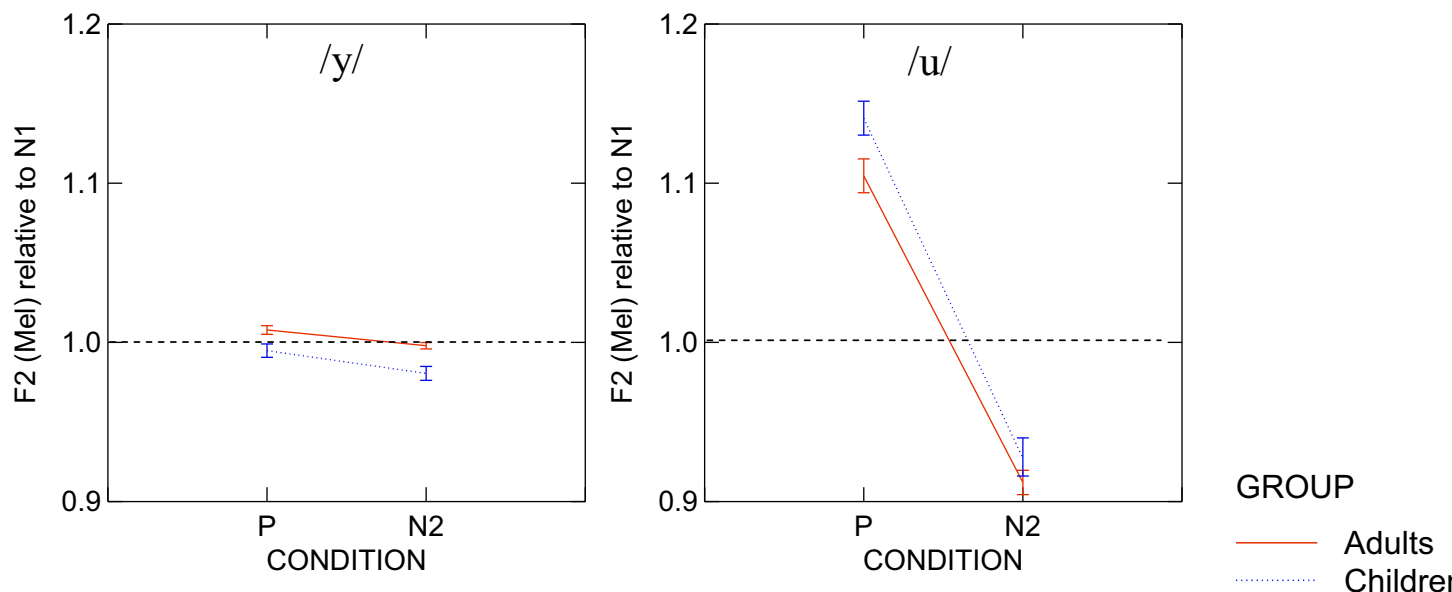

FIGURE 5. F2 values (in Mel) relative to the normal pre-perturbed condition N1, averaged across speaker groups, for the perturbed condition $\mathrm{P}$ and the normal post-perturbed condition N2. Adult data correspond to the solid red line and child data to the dotted blue line. Data are shown separately for $/ \mathrm{y} /$ (left panel) and /u/ (right panel).

Average F3 values relative to the N1 condition are shown for the perturbed $\mathrm{P}$ and the post-perturbed N2 conditions, for children and adults in Figure 6. Recall that F3 was increased upon insertion of the tube, according to the simulations presented in Figures 1 and 2. A significant effect of condition is found for the vowel $/ \mathrm{y} /$ $(\mathrm{F}(1,18)=59.38 ; \mathrm{p}<.05)$, with $\mathrm{F} 3$ being higher in the $\mathrm{P}$ condition than in the $\mathrm{N} 1$ (values greater than 1) and $\mathrm{N} 2$ conditions for adults. Thus, adults might not have totally compensated for the lip-tube. Figure 6 suggests that children were more successful than adults in decreasing $\mathrm{F} 3$ for $/ \mathrm{y} /$ in the $\mathrm{P}$ condition (F3 values are significantly lower for children than for adults). Some F3 changes are observed for / $\mathrm{u}$ (especially an after-effect for the children group, revealed by F3 ratios in N2 lower than 1), likely triggered by compensation strategies targeting other formants.
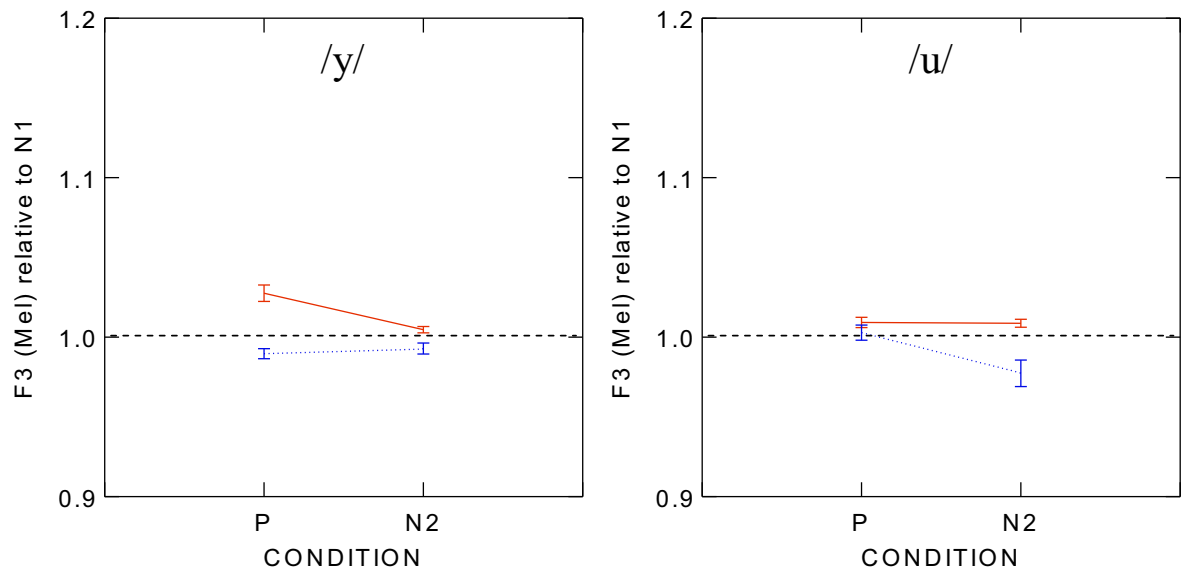

GROUP

Adults Children

FIGURE 6. F3 values (in Mel) relative to the normal pre-perturbed condition N1, averaged across speaker groups, for the perturbed condition $\mathrm{P}$ and the normal post-perturbed condition N2. Adult data correspond to the solid red line and child data to the dotted blue line. Data are shown separately for $/ \mathrm{y} /$ (left panel) and /u/ (right panel).

The acoustic patterns depicted in Figures 4 to 6 may be related to various articulatory strategies. We will focus only on the front-back position of the tongue in this paper. Data are presented in Figure 7, for both vowels, conditions, and speaker groups. In this graph, a value greater than 0 corresponds to a more backward tongue position than in the N1 condition,

An examination of Figure 7 reveals group differences in the articulatory positions $(\mathrm{F}(1,18)=24.56 ; \mathrm{p}<.05)$. For adults, the stability of the relative front-back position of the tongue across the perturbed condition $\mathrm{P}$ and the postperturbation condition N2 suggests that the participants produced a more back position of the tongue in order to compensate for the insertion of the tube. During the perturbation trials, this position was integrated into the 
articulatory-acoustic models and the new articulatory strategy was retained after removal of the tube (N2). For the child group, this pattern is observable only for the vowel $/ \mathrm{u} /$. For $/ \mathrm{y} /$, on the contrary, despite a more backward position of the tongue in the $\mathrm{P}$ condition, the tongue remains in a position close to that in $\mathrm{N} 1$ after removal of the tube (N2 condition).
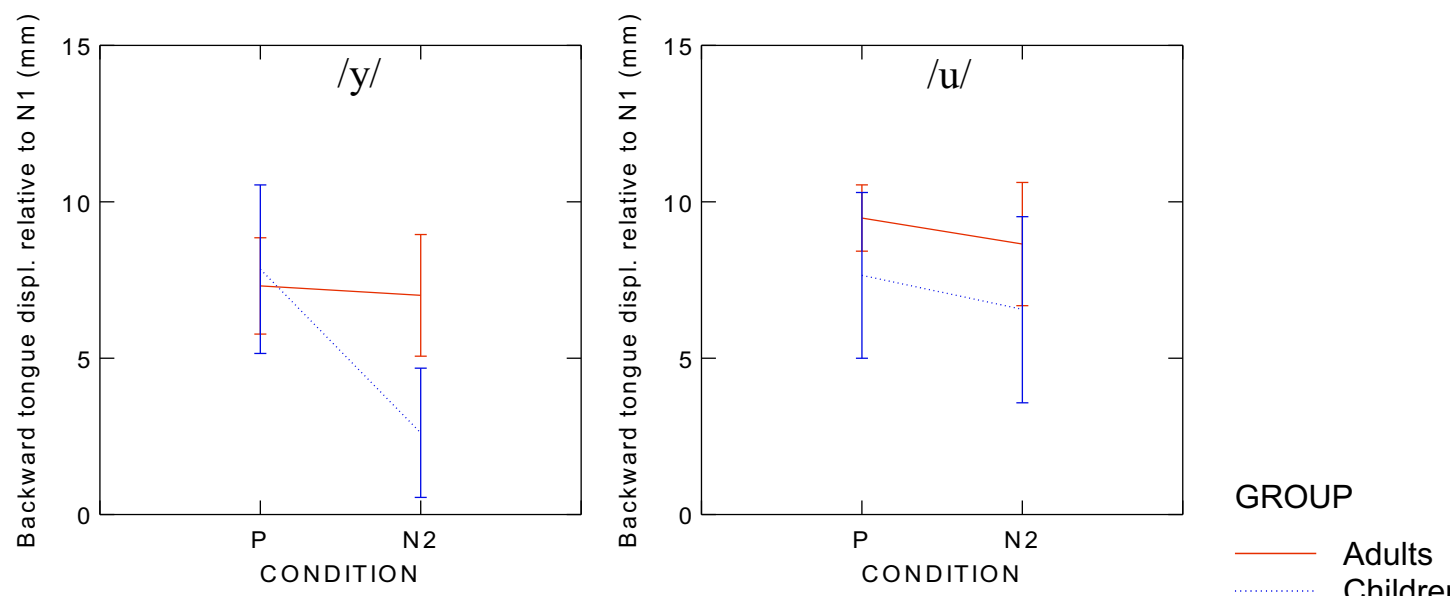

FIGURE 7. Backward tongue displacement relative to the normal pre-perturbed condition N1, averaged across speakers, for the perturbed condition $\mathrm{P}$ and the normal post-perturbed condition N2. Adult data correspond to the solid red line and child data correspond to the dotted blue line. Data are shown separately for /y/ (left panel) and /u/ (right panel).

\section{DISCUSSION}

In both groups, the perturbation induced changes in tongue positioning that are consistent with a reduction of the impact of the perturbation on the spectral characteristics of the vowels. This result provides support to the hypothesis that in children and in adults, compensation strategies are predominantly guided by the search for auditory goals, and that the acoustic feedback is required for the subjects to build-up these strategies.

The perceptual tests show that, in the perturbed condition, both speaker groups produced vowels that were associated with a lower perceptual score than in the normal pre-perturbed condition. This pattern suggests that, perceptually, the compensatory strategies were not completely successful. In addition, after removal of the tube, perceptual scores were higher than in the normal pre-perturbed condition, suggesting a considerable after-effect. After-effects are classically considered as evidence for learning. Such an effect was already observed in our former lip-tube experiment with children (Ménard et al, 2008). In this study, we considered this effect to be in contradiction with our other finding showing that children did not select any specific compensatory strategy at the end of the training phase. The articulatory data collected in the current study shed some light on this intriguing contradiction.

When averaging across the different repetitions in the $\mathrm{P}$ condition, children and adults achieved a more backward articulation for both vowels. In the post-perturbed condition, this global postural change is clearly maintained in adults, while it is maintained only for $/ \mathrm{u} /$ in children. This difference between adult and children suggest that different mechanisms could underlie the construction of compensatory strategies: in adults the backward articulatory readjustment seems to be memorized and generalized for both vowels, which is consistent with the use an internal model; this mechanism does not seem to operate in 4-year-old children.

The acoustic data show that the backward tongue displacement is more efficient for children in /y/ (F2 and F3 in $\mathrm{P}$ condition are closer to those in $\mathrm{N} 1$ condition) and more efficient for adults in $/ \mathrm{u} / \mathrm{F} 2$ in $\mathrm{P}$ condition closer to the value in N1 condition). This might be due to differences in the articulatory-to-acoustic relations associated with morphological differences across age (see Ménard et al., 2007). This hypothesis deserves further investigation. In any case, this result suggests that, as compared to adults, 4-year-old children do not show any obvious intrinsic limitation to deal with the lip perturbation. Interestingly, at the acoustic level, after-effects are observed for both vowels in adults and in children. For $/ y /$, this observation is not consistent with the articulatory data which show no after effect. This inconsistency could be due to the existence in children of other compensatory maneuvers that did not induce any change in the front/back tongue positioning. It could also be due to an increase of lip rounding in N2 condition due to an increase of the overall force in the orbicularis oris generated during the $\mathrm{P}$ condition by the presence of the tube inserted between the lips. 
Overall, at this stage of the study, our interpretation of the data is as follows:

- children and adults use their auditory feedback to build-up compensatory strategies aiming at reaching the auditory goals of their speech under normal conditions;

- adults rely on internal model to elaborate the compensatory strategies and to memorize them in a general way;

- $\quad$ children also elaborate compensatory maneuvers but they do not memorize them as systematically as adults do;

- the articulatory data show that in adult, after-effects are associated with the maintenance of the articulatory strategies built-up in the P condition; in children, the link between after-effects and changes in tongue position does not seem to apply systematically; a part of this after-effect could be associated with other factors such as lip muscles pre-settings physically induced by the insertion of the lip-tube in the $\mathrm{P}$ condition.

Overall, these results suggest that, if they exist, internal models in children do have the same capacities as in adults to systematically guide the Central Nervous System towards an efficient compensatory strategy and to store it. This is consistent with the trial-and-error strategy observed in our former study (Ménard et al., 2008) during the training phase. This has to be confirmed by further investigation, in particular by a careful analysis of the articulatory and acoustic changes provided by each subject during the $\mathrm{P}$ condition. A systematic and continuous improvement of the spectral patterns, similar to the one observed in Savariaux et al. (1995), associated with clear articulatory aftereffect, will provide support for the hypothesis of the use of an internal model; a more erratic variation, similar to the one observed in Ménard et al (2008), associated with non systematic articulatory after-effect, will speak for a trialand-error strategy making little use of internal models.

\section{ACKNOWLEDGMENTS}

This work was supported by an SSHRC grant and an NSERC grant. Thanks to Zofia Laubitz for copyediting the paper. The authors wish to thank Christine Turgeon, Joseph Perkell, and Mark Tiede for their insightful comments on an earlier version.

\section{REFERENCES}

Aubin, J. (2005). Effets acoustiques et articulatoires de perturbations labiales sur la parole des enfants et des adultes (Acoustic and articulatory effects of labial perturbations on adult and child speech), MA thesis, Montreal : Université du Québec à Montréal.

Li, M., Kambhamettu, C., and Stone, M. (2003). "EdgeTrak, a program for band-edge extraction and its applications," Sixth IASTED International Conference on Computers, Graphics and Imaging, Honolulu, HI, August 13-15.

Lindblom, B. and Sundberg, J. (1971). "Acoustical consequences of lip, tongue, jaw and larynx movement," J. Acoust. Soc. Am. 50, 1166-1179.

Ménard, L., Aubin, J., Thibeault, M., and Richard, G. (2012). "Comparing tongue shapes and positions with ultrasound imaging: a validation experiment using an articulatory model, " Folia Phoniatrica et Logopaedica, 64, 64-72.

Ménard, L., Perrier, P., Savariaux, C., Aubin, J., and Thibeault, M. (2008). "Compensation strategies for a lip-tube perturbation of French [u] : an acoustic and perceptual study of 4-year-old children," J. Acoust. Soc. Am., 124, 1192-1206.

Ménard, L., Schwartz, J.-L., Boë, L.-J., and Aubin, J. (2007). "Production-perception relationships during vocal tract growth for French vowels: analysis of real data and simulations with an articulatory model," Journal of Phonetics, 35 (1), 1-19.

Savariaux, C., Perrier, P., Orliaguet, J.-P., and Schwartz, J.-L. (1999). "Compensation strategies for the perturbation of French [u] using a lip tube. II. Perceptual analysis,” J. Acoust. Soc. Am. 106, 381-393.

Savariaux, C., Perrier, P., and Orliaguet, J.-P. (1995). "Compensation strategies for the perturbation of the rounded vowel [u] using a lip-tube: A study of the control space in speech production," J. Acoust. Soc. Am. 98, 2428-2442.

Wolpert, D.M., Ghahramani, Z., Daniel M., and Flanagan, J.R. (2001). "Perspectives and problems in motor learning," TRENDS in Cognitive Sciences, 5(11), 487-494. 